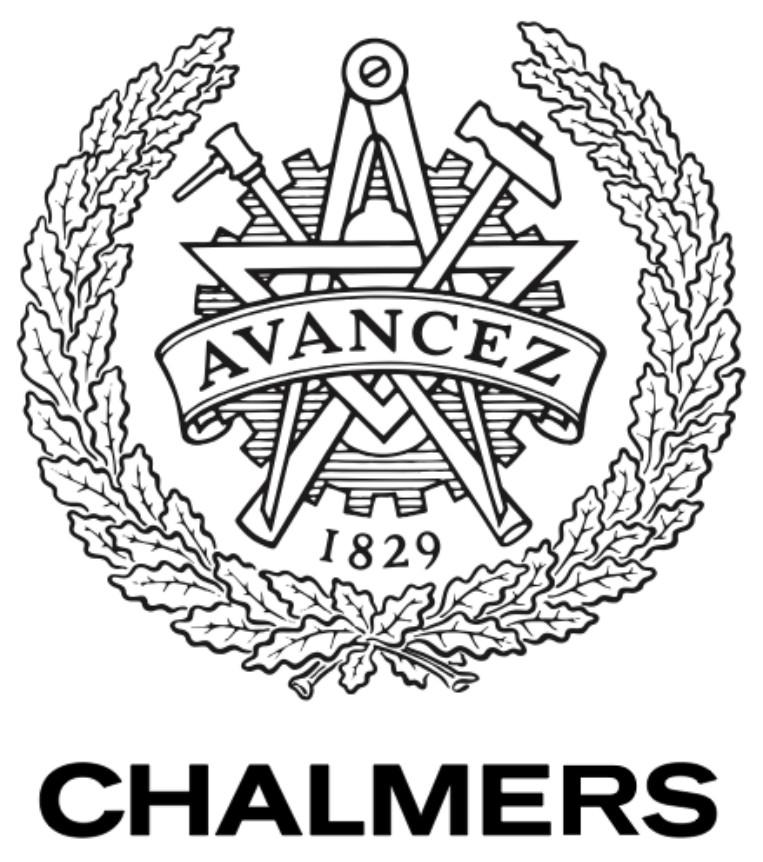

Chalmers Publication Library

\title{
All Aboard the Robotic Road Train
}

This document has been downloaded from Chalmers Publication Library (CPL). It is the author's version of a work that was accepted for publication in:

leee Spectrum (ISSN: 0018-9235)

Citation for the published paper:

Coelingh, E. ; Solyom, S. (2012) "All Aboard the Robotic Road Train". Ieee Spectrum, vol. 49(11), pp. 34-39.

Downloaded from: http://publications.lib.chalmers.se/publication/168996

Notice: Changes introduced as a result of publishing processes such as copy-editing and formatting may not be reflected in this document. For a definitive version of this work, please refer to the published source. Please note that access to the published version might require a subscription. 


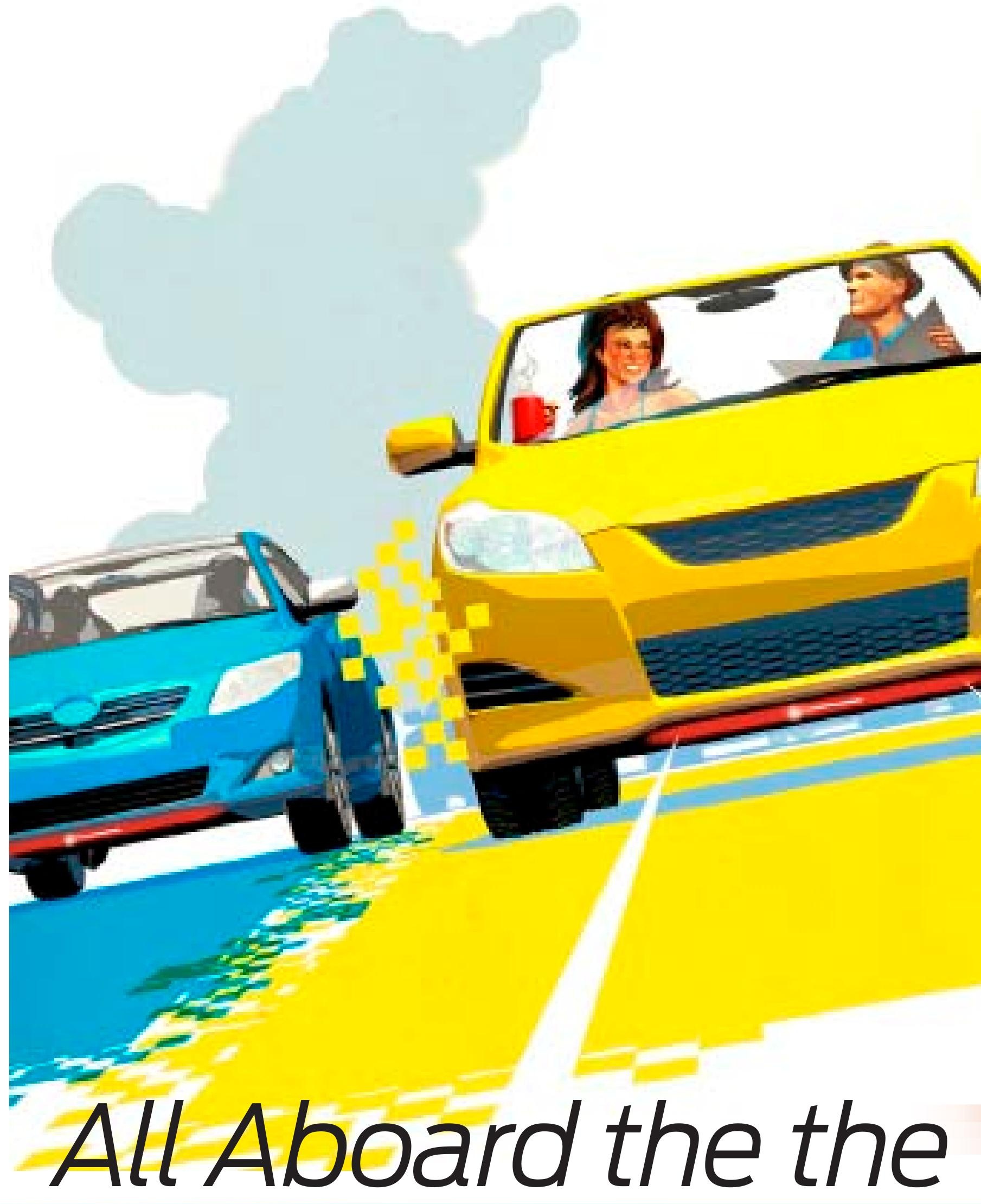

SIT BACK, RELAX, AND LET THE CAR IN FRONT OF YOU DO THE DRIVING 


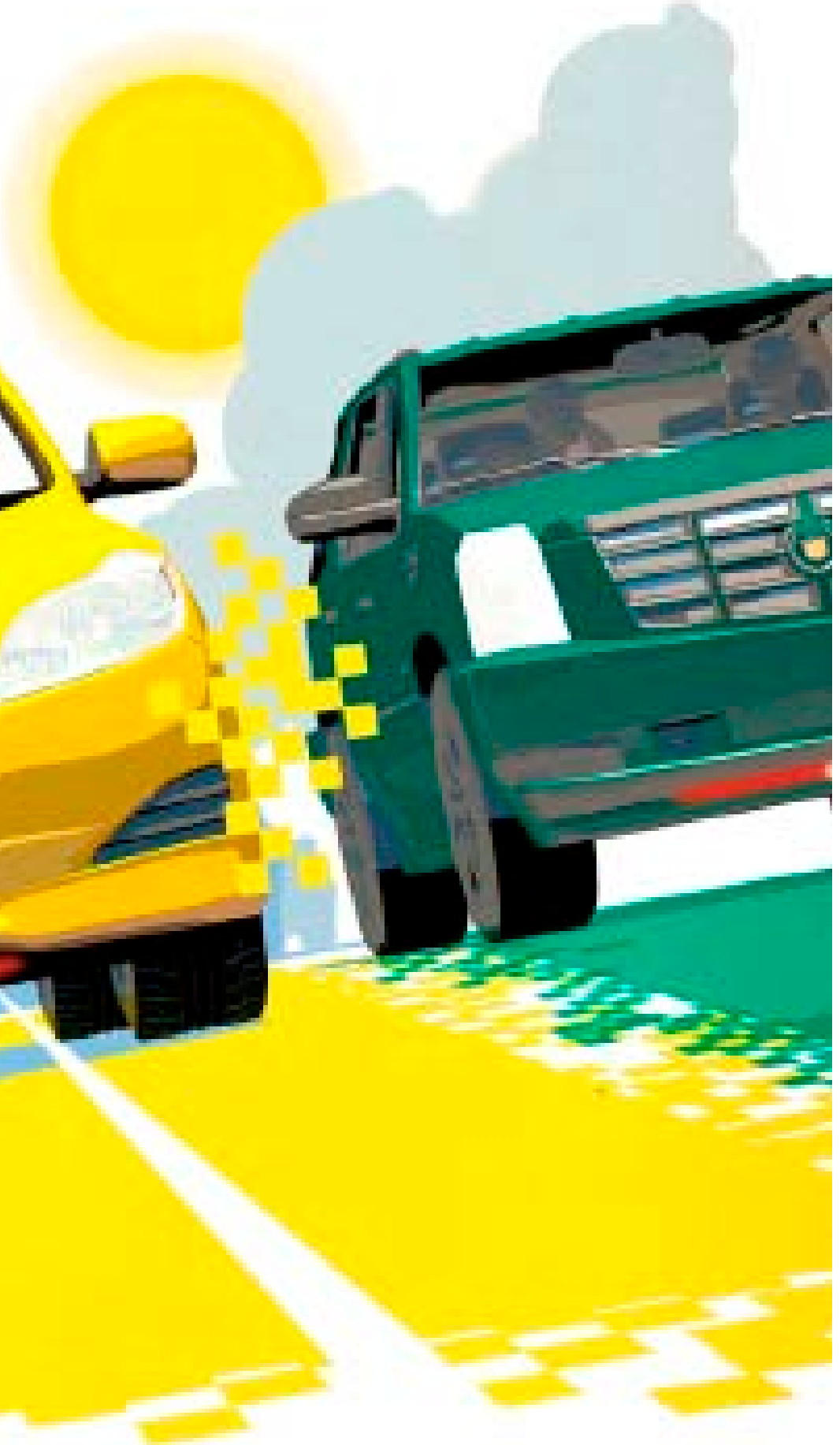

N CAR COMMERCIALS EVERY ROAD is clear and curvy, every vista is framed by mountains and the sea, and every driver is relaxed and in the moment. In real life, though, driving is often as much a pain as it is a pleasure-a car, once a symbol of independence, is now perhaps the last place where you can't use your smartphone. Even when the roads aren't clogged, you must be constantly alert because, let's face it, too many other drivers are inattentive or downright maniacal (characteristics that never apply to you, of course!). Public transportation has its own drawbacks: Buses and trains don't start at your home and don't end at your destination, nor do they leave just when you'd like or even guarantee you a seat.

To get the best of both worlds, we could teach our cars to work together, as closely grouped cyclists do in a peloton. The lead car could be entrusted to a professional driver to whom the other drivers would of course each pay a small fee; all the other cars would follow it automatically. The cars would all use networked communications coupled with the optical or electromagnetic sensors already installed in some luxury cars to avoid head-on collision, stay in the proper lane, and brake in case of emergency. These systems have been developed at great expense to provide active safety, as distinguished from the passive kind afforded by seatbelts. But this investment, having been made, can now be exploited for other things-like allowing you to relax and read the paper. If only we'd let them.

Active systems are improving at a splendid rate. Adaptive cruise control, for example, maintains a car's speed while using radar or lidar to keep a safe distance from the car in front of it, thus automating much of the braking and accelerating. The latest generation of this system can follow a lead car from highway speed to a stop and then resume automatically when that car drives away. Soon the system will get additional data from vision sensors and digitized maps and additional support for the steering, allowing it to slow down in curves.
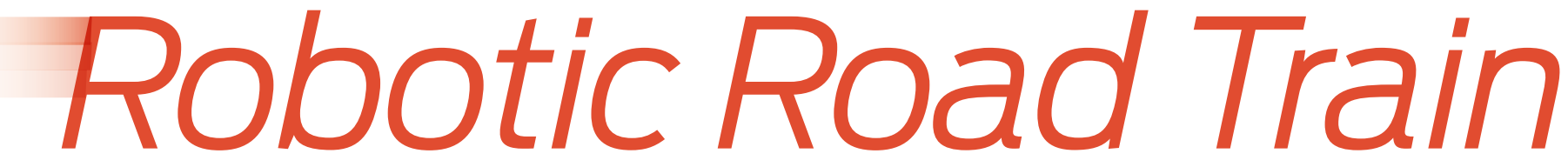


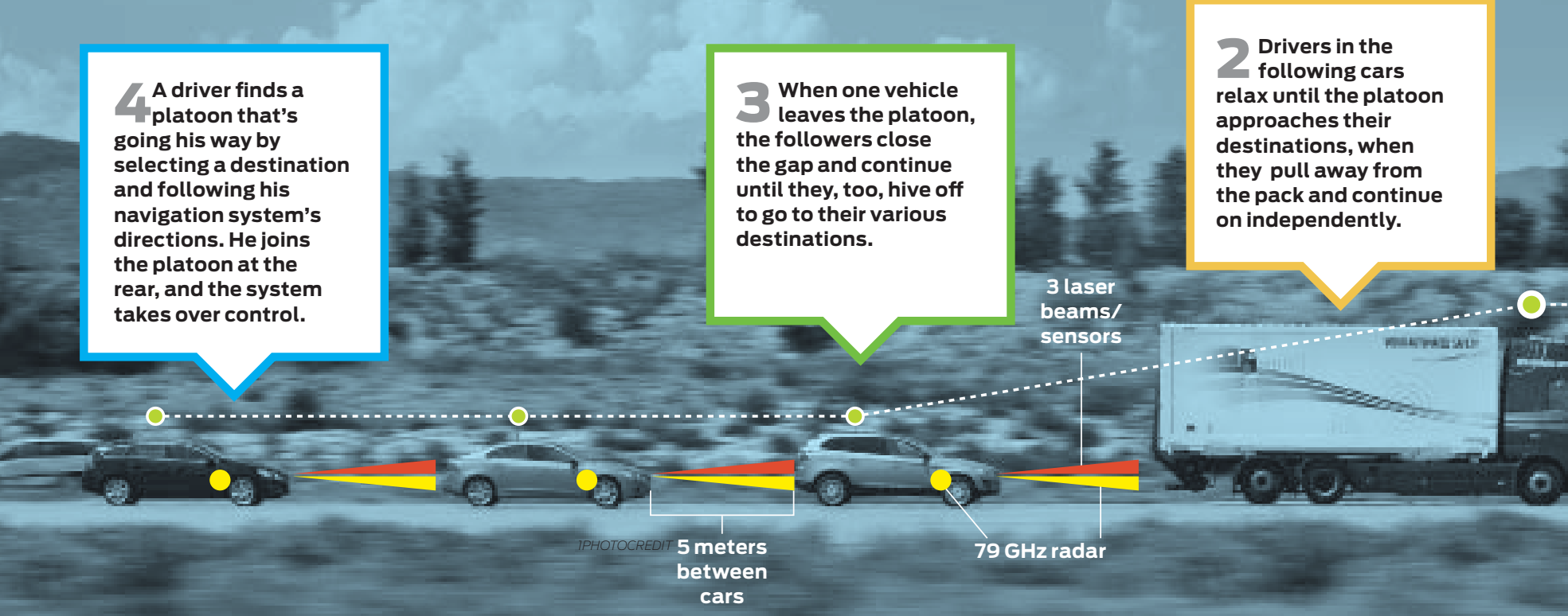

RUBBERNECKING'S OKAY for drivers in this fivemember platoon, led by a truck, one of many tests of the SARTRE project in Sweden and Spain. Guidelines allow for up to 10 members-more, and it becomes difficult for outsiders to see exit ramps from the highway.

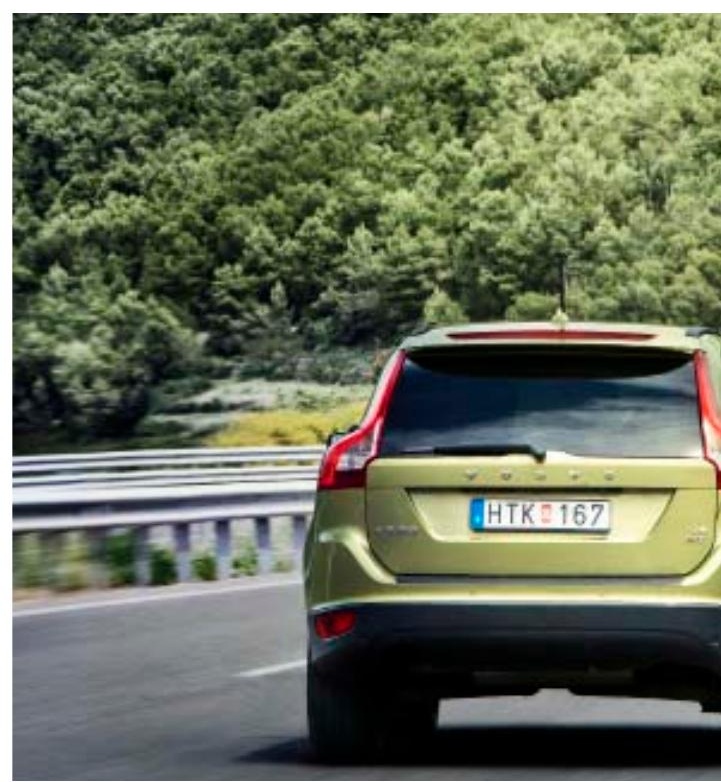

safe. Just to verify Volvo's pedestrian detection with fully automatic braking had required driving more than $500000 \mathrm{~km}$ and collecting more than 3 terabytes of data (mostly to confirm that the risk of inadvertent braking would be acceptably low). To ensure that an autonomous car would almost never make severe mistakes, no matter what the weather and traffic situations might be, would be vastly harder. We concluded that such a fully autonomous car would, for now, be a step too far.

To advance the state of the art, we decided to come up with an intermediate goal. We quickly focused on the concept of platooning, which gives the lead driver responsibility only in the middle part of the trip. In this scheme, the person behind the wheel of each of the following cars does all the work at tem to give a car full autonomy. We realized that the chief problem lay in making sure that such a system would be utterly 
driver leading them, who is aided by the most advanced collision-avoidance technologies available.

The main problem with the PATH system was that it couldn't be easily extended to other roads. It's a long, expensive job to change the roads, and it's not likely to happen when there are no vehicles to use them. Nor will carmakers want to build vehicles that use magnetic guidance if there aren't many roads to drive them on. We need a way for autonomous cars to run on existing roads.

To work out such a system, the European Community funded the Safe Road Trains for the Environment (SARTRE) project, which started in 2009 and ended September 2012. The participants included Britain's Ricardo, which coordinated the project; Spain's IDIADA Automotive Technology and Tecnalia Research \& Innovation; Germany's Institut für Kraftfahrzeuge Aachen; and the SP Technical Research Institute of Sweden, Volvo Car Corp., and Volvo Technology. Our strategy with SARTRE was to rely, to the extent possible, on technologies already available in production cars, rather than on exotic and largely unproven new technologies. And to tame the problem of verification, we not only entrusted the lead vehicle to a professional driver who could deal with exceptional situations but also limited the automated driving to roads that had at least two lanes going in the same direction. On such roads the traffic situations usually are not complex, and it's easy for faster-moving cars to pass a road train.

We put the heaviest vehicles up front to reduce the risk of collisions during emergency braking, because a heavy vehicle decelerates more slowly than a lighter one. We programmed each vehicle to align laterally with the leader and to maintain a proper distance from the car directly in front of it; that way, if the leader changes lanes or engages in evasive maneuvers, all the other cars will follow the path it blazes while remaining in their queue. We also calculated that, at a maximum speed of 90 kilometers per hour ( 56 miles per hour), the platoon could have no more than about 10 vehicles or its close-packed formation would block access to exit ramps. Drivers would join a platoon either on the spot, by means of an electronic request or by booking a place in advance. Highway driving was our sole interest, because it is the least complicated kind, lacking intersections, traffic lights, bicyclists, and so on.

NONE OF OUR REQUIREMENTS are outlandish, yet they do define the problem narrowly enough to make it solvable with existing technology. We implemented a limited number of critical platoon scenarios representing how the cars interactfor instance, Join Platoon, Maintain Platoon, Leave Platoon, and Dissolve Platoon. Anyone joining the platoon would normally do so at the rear, but we could allow for someone to join in the middle by enabling the controlling system to tell one member of the pack and those following it to slow down, thus opening up a space.

These transitions to and from automatic driving are cru- cial because a driver should never be unsure whether he or the lead car is in control. To avoid any such uncertainty, we have chosen to coordinate these transitions with a user interface that, although new, will still be familiar to drivers because it
DRIVER DISTRACTION, a side-effect of our sometimes excessively linkedin world, is here not a bug, but a feature. Within a few minutes of joining a latoon, drivers tend to kick back and take their attention from the road. is based on the existing one in active cruise control systems.

To join from the rear, a driver would send a request to the lead vehicle, get confirmation, approach the platoon from behind, and then put the car into semiautomatic mode, in which braking and accelerating is automatic and the steering is still manual. This ensures that the driver will pay full attention to traffic in case anything unusual happens. Only when the car is locked into the determined following distance does lateral control pass to the automatic system. An indication of the change appears on the car's display, accompanied by a voice message, letting the driver know that he can release the steering wheel, lean back, and just enjoy the ride.

SARTRE designed a prototype system consisting of a lead vehicle, a following truck, and three cars-a Volvo S6o, a V6o, and an XC60, modified to allow for fully automatic driving. We started development in 2009 and began testing vehicles in the summer of 2010, mostly at Volvo's Hällered proving ground in Sweden. We performed tests of fuel consumption at IDIADA in Spain and a test drive on $200 \mathrm{~km}$ of public roads, also in Spain.

Within a car, the system communicates with the power train, brakes, and steering through Volvo's standard adaptive cruise control and lane-keeping guidance. It measures the distance between cars with two other standard systems: a short-range system of three laser beams, which measures distances of up to 8 meters ahead, and the 76 -gigahertz radar in 
the active cruise control, which measures the ranges of objects up to 200 meters ahead.

The side-to-side movement of the car up ahead is monitored by a forward-looking camera that's used in our current production vehicles to detect pedestrians and recognize traffic signs.

To keep the platoon and its lead driver apprised of traffic in neighboring lanes, the cars also come with two rear- and side-looking radars, also at $76 \mathrm{GHz}$. Not only do the radars watch out for swerving cars in the next lane, they also help the system track and then accommodate the entry of any car that may join the platoon.

We noticed a potential glitch during trials on our test track in Sweden, where the sun shines from a low angle. Every time we rounded a particular curve, the sun would blind our camera, making the car lose sight of the vehicle in front and suddenly veer out of its lane-a very unpleasant feeling! We tried several backup plans and finally hit on the solution. We took the lead vehicle's estimation of the curvature of the path it is taking and the radar's measurement of the azimuth anglethe horizontal angle between our own car's radar sensor and the car directly in front of us. This technique is normally rather imprecise, as the radar reflections don't necessarily come from the center of the preceding vehicle; sometimes they come from the corners or even the side. To assure ourselves that it worked well, we put tape on the windshields to simulate camera blockage and drove many laps. I remember sitting in the vehicle for about two hours, quite comfortably listening to a football match on the radio. The road train was finally working as intended.

The new, custom-made equipment we added to make the system fully automatic includes an interface that allows the cars (though not their occupants) to communicate. The system could also feed real-time video from a camera in the lead vehicle to people in the platoon to make them more comfortable with their short following distances. A touch screen displays the status of each car (joining, maintaining, or leaving the platoon), and vibrators in the car seat provide warning whenever the driver has to take immediate action-for instance, when part of the platoon has to be dissolved because another vehicle has wormed its way into it.

We also incorporated a prototype wireless system, based on the 5.9-GHz IEEE 802.11p Wi-Fi standard, to allow direct data links among all the vehicles in the platoon. The $802.11 \mathrm{p}$ standard was originally devised to allow vehicles to communicate automatically with roadside beacons, to collect tolls, for instance, and with other vehicles to avoid accidents.

Overall control is distributed throughout the platoon. The lead vehicle contains the "organizational assistant," which keeps track of the platoon as a whole-the number of vehicles, their order, speed, gap size, and so on. It gets this information through direct communication links to all the other vehicles. The organizational assistant sends out a series of set points describing longitudinal acceleration and the curvature of the path that is driven-not that of the road. It's as if the lead car were dropping bread crumbs for the others to follow. Each follower contains control units that strive to follow these set points while keeping a minimum gap and a maximum lateral offset with respect to the vehicle directly in front. The lead vehicle tracks these separation distances, and it can command a follower to open up a larger gap should another vehicle try to merge.

Note that the lead vehicle of the platoon is the one they're following in a real sense-they merely sense and avoid the other cars. If, for example, the lead driver suddenly slams on the brakes, the communication system commands all the other vehicles to brake, decelerating all of them at once so that none collides with the car in front of it. Without that communication link, there would be a small delay, and the delays would add up as they progress through the chain; the last vehicle would thus have to brake much faster and harder than the first one. Similar effects-they're called string instabilities-would occur if the leader's decisions on steering were not also immediately communicated to all the followers.

We are still validating the prototype, driving at different speeds and with different amounts of space between the vehicles. We find that though shorter separations decrease aerodynamic drag, they also require stiffer control, which often means a heavier robotic "foot" on the accelerator or the brake, which can undo some of the fuel savings. On the other hand, our tests indicate that drivers prefer the stiffer control; it seems that they feel secure, as if their car were driving on rails. At first, they tend to be alert and ready to take over at any time, but within a few minutes they relax and take their attention from the road.

A FEW PROBLEMS REMAIN. For instance, during our winter tests in Sweden, we found that trailing only 5 meters behind a heavy vehicle meant getting a windshield full of salty spray and gravel. We had to clean the windshield constantly to keep the forward-looking camera unblocked; sometimes it felt as if we were consuming more washer fluid than gasoline. Also, the gravel dinged our car quite a bit. Conclusion: Although 5 meters may be aerodynamically attractive, we may have to increase it sometimes.

In the end, though, the biggest challenges will probably be legal ones. Right now driving on autopilot isn't allowed in most areas in Europe and North America, and there is no consensus on who would be responsible for any accident.

What we have learned is that there is a practical way station on the road to fully self-driving cars, and that today's vehicles already have most of the technology they need to get there. Airplanes have been moving among similar way stations for decades now, using flight control systems, coupled with evercloser coordination with ground control stations, to supplement rather than replace the pilots on board.

If on land, as in the air, semiautomated systems can save fuel and perhaps lives, and if they give drivers freedom to spend their time as they wish, then it will be a clear gain. And experience with such systems will undoubtedly teach us much of what we need to know to devise fully autonomous drivers that do not know fatigue, impatience, or the temptation of skirting the dictates of law and decorum on the road.

GOST YOUR COMMENTS online at spectrum.ieee.org/channelname/slug/online-headline-here 